\title{
Trabecular Adenocarcinoma
}

National Cancer Institute

\section{Source}

National Cancer Institute. Trabecular Adenocarcinoma. NCI Thesaurus. Code C4068.

A malignant epithelial neoplasm characterized by the presence of a trabecular glandular architectural pattern. 